\title{
Intrinsic Defect-Rich Hierarchically Porous Carbon Architectures Enabling Enhanced Capture and Catalytic Conversion of Polysulfides
}

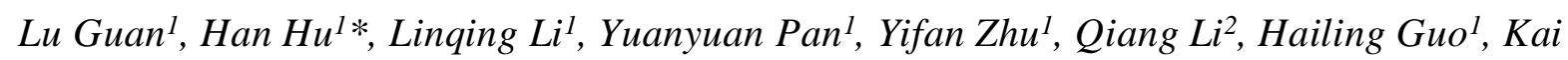
Wang ${ }^{3}$, Yunchun Huang ${ }^{1}$, Mengdi Zhang ${ }^{1}$, Yingchun Yan ${ }^{1}$, Zhongtao Li ${ }^{1}$, Xiaoling Teng ${ }^{1}$, Junwei Yang ${ }^{1}$, Jiazhi Xiao ${ }^{1}$, Yunlong Zhang ${ }^{1}$, Xiaoshan Wang ${ }^{1}$ and Mingbo Wu ${ }^{1 *}$

${ }^{1}$ State Key Laboratory of Heavy Oil Processing, Institute of New Energy, College of Chemical Engineering, China University of Petroleum (East China), Qingdao 266580, China ${ }^{2}$ College of Physics Science, Qingdao University, Qingdao, 266071, China.

${ }^{3}$ College of Electrical Engineering, Qingdao University, Qingdao, 266071, China. 


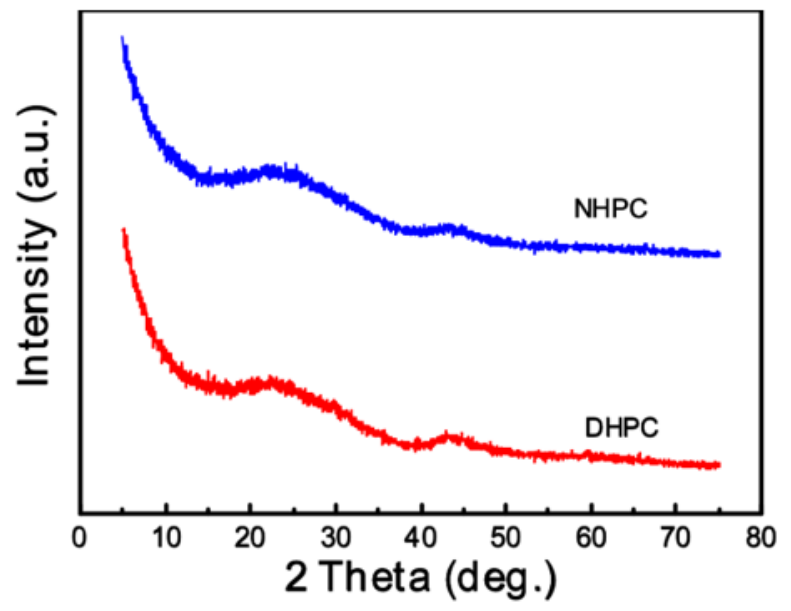

Figure S1. XRD patterns of NHPCs and DHPCs. 

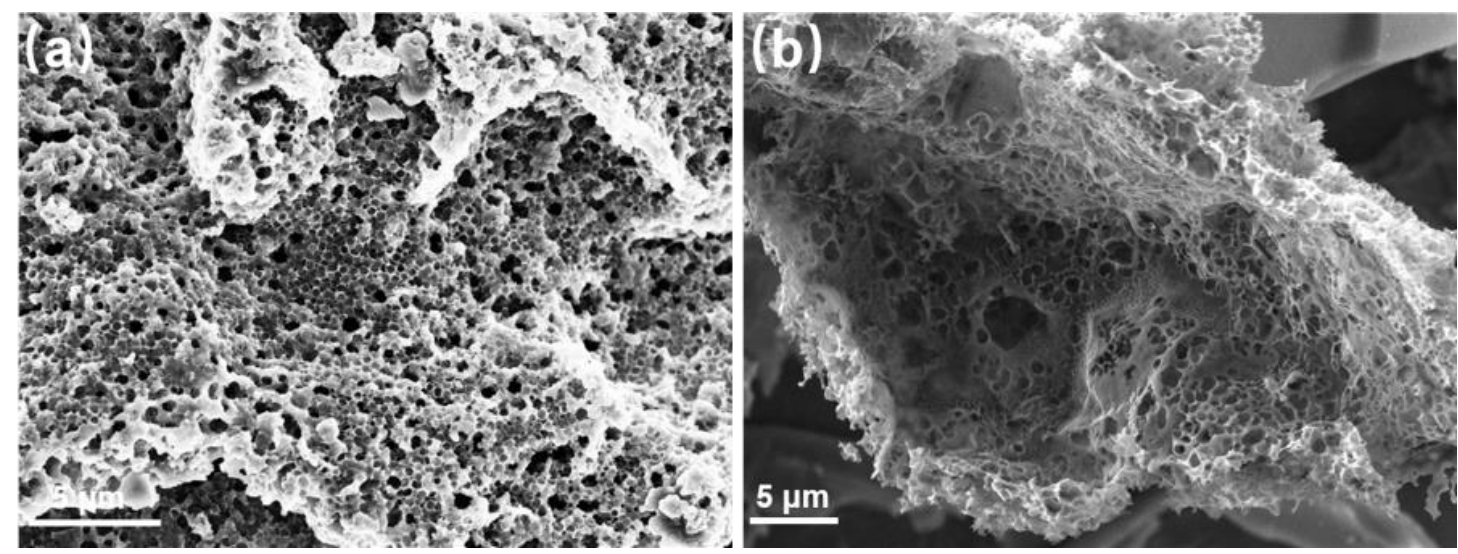

Figure S2. SEM images of (a) NHPCs and (b) DHPCs at low magnification. 
(a)

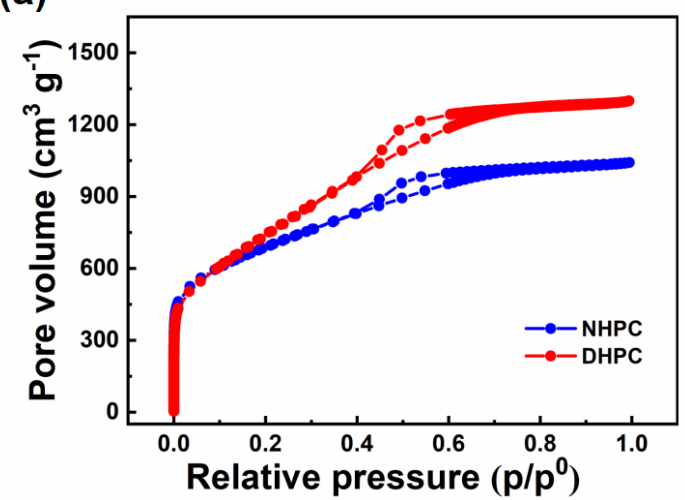

(b)

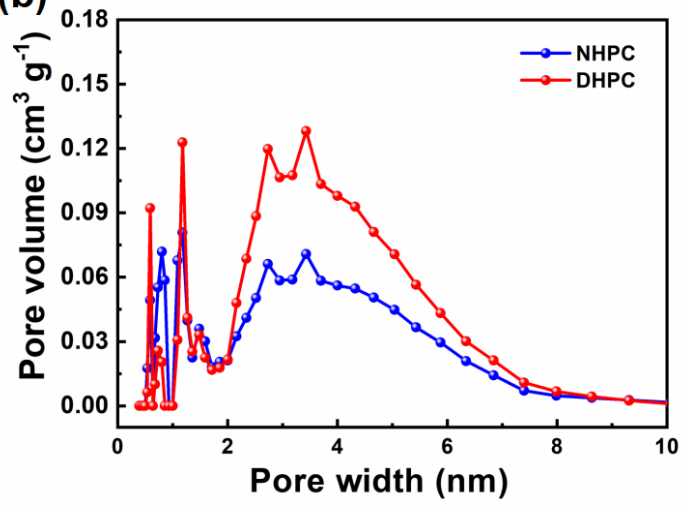

Figure S3. (a) $\mathrm{N}_{2}$ sorption isotherms and (b) pore size distributions of NHPCs and DHPCs.

Table S1. Detailed pore structure of NHPCs and DHPCs

\begin{tabular}{|c|c|c|c|c|c|c|}
\hline Samples & $\begin{array}{c}D_{\text {ap }} \\
{[\mathrm{nm}]}\end{array}$ & $\begin{array}{c}S_{\text {BET }} \\
{\left[\mathrm{m}^{2} \mathrm{~g}^{-1}\right]}\end{array}$ & $\begin{array}{c}S_{\text {mic }} \\
{\left[\mathrm{m}^{2} \mathrm{~g}^{-1}\right]}\end{array}$ & $\begin{array}{c}V_{\mathrm{t}} \\
{\left[\mathrm{cm}^{3} \mathrm{~g}^{-1}\right]}\end{array}$ & $\begin{array}{c}V_{\mathrm{mic}} \\
{\left[\mathrm{cm}^{3} \mathrm{~g}^{-1}\right]}\end{array}$ & $\begin{array}{c}V_{\text {mic }} / V_{\mathrm{t}} \\
{[\%]}\end{array}$ \\
\hline NHPC & 2.68 & 2405 & 2192 & 1.61 & 1.36 & 84.47 \\
\hline DHPC & 2.90 & 2769 & 2426 & 2.01 & 1.64 & 81.59 \\
\hline
\end{tabular}



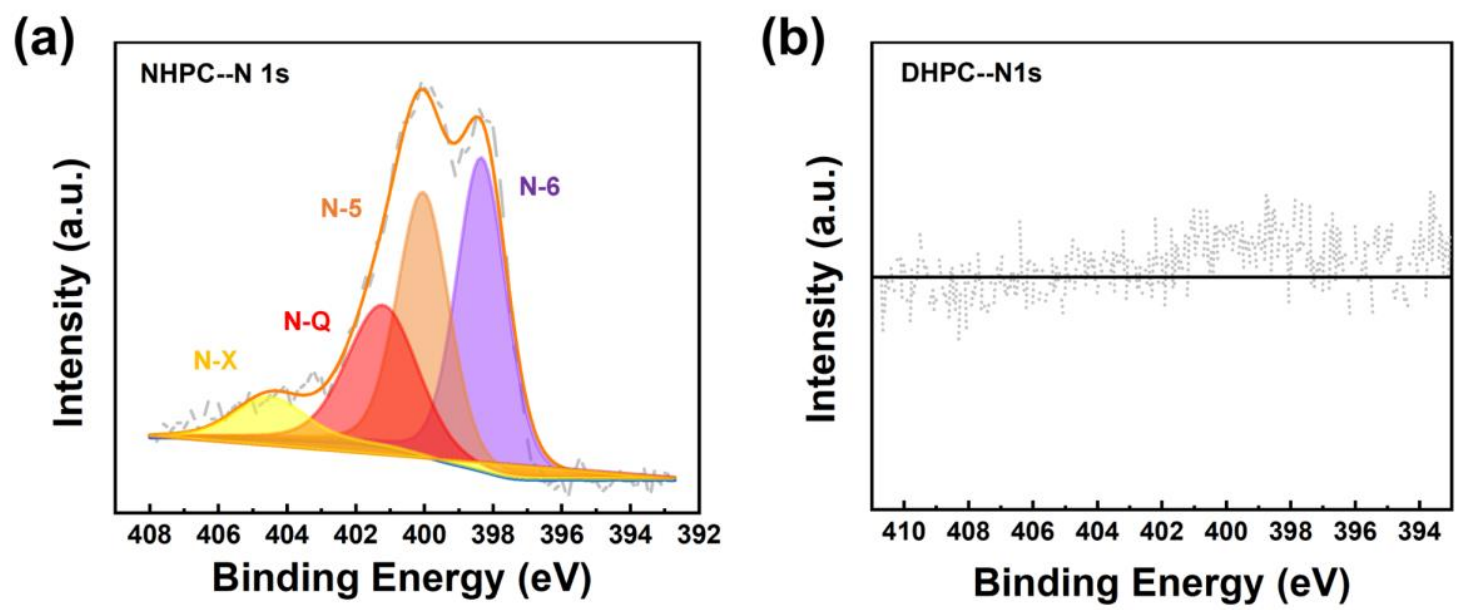

Figure S4. N1s spectrum of (a) NHPCs and (b) DHPCs.

Table S2. Elemental composition of NHPCs and DHPCs

\begin{tabular}{cccc}
\hline & \multicolumn{3}{c}{ Element (atomic ratio \%) } \\
\cline { 2 - 4 } Samples & $\mathrm{C}$ & $\mathrm{N}$ & $\mathrm{O}$ \\
\hline NHPC & 84.19 & 7.99 & 7.82 \\
DHPC & 87.25 & 0 & 12.75 \\
\hline
\end{tabular}

Table S3. Electrical conductivity of NHPCs and DHPCs

\begin{tabular}{cc}
\hline Samples & Conductivity $(\mathrm{S} / \mathrm{m})$ \\
\hline NHPC & 3.62 \\
DHPC & 6.17 \\
\hline
\end{tabular}



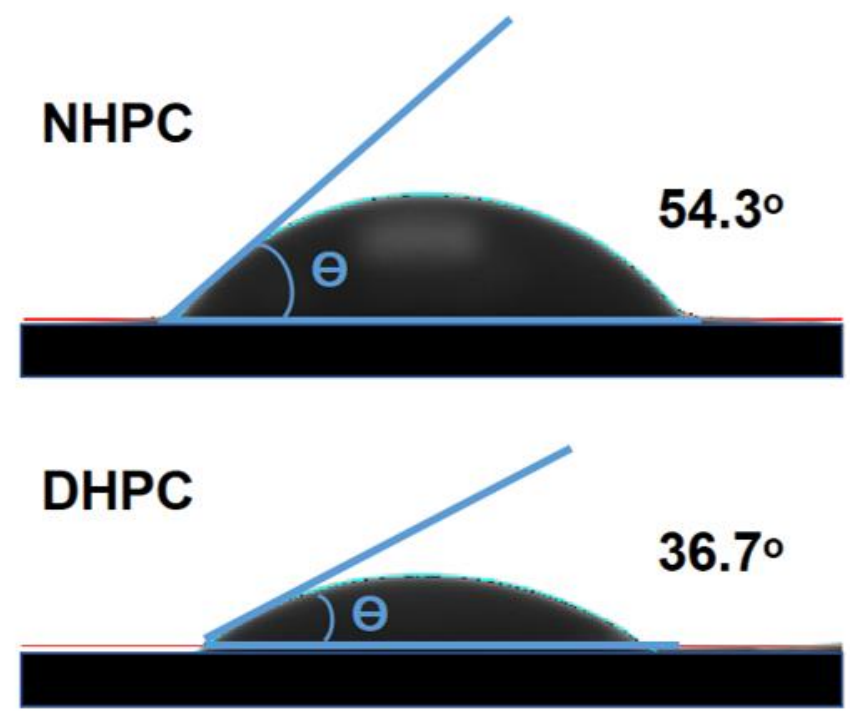

Figure S5. Water contact angle measurement of NHPCs and DHPCs. 


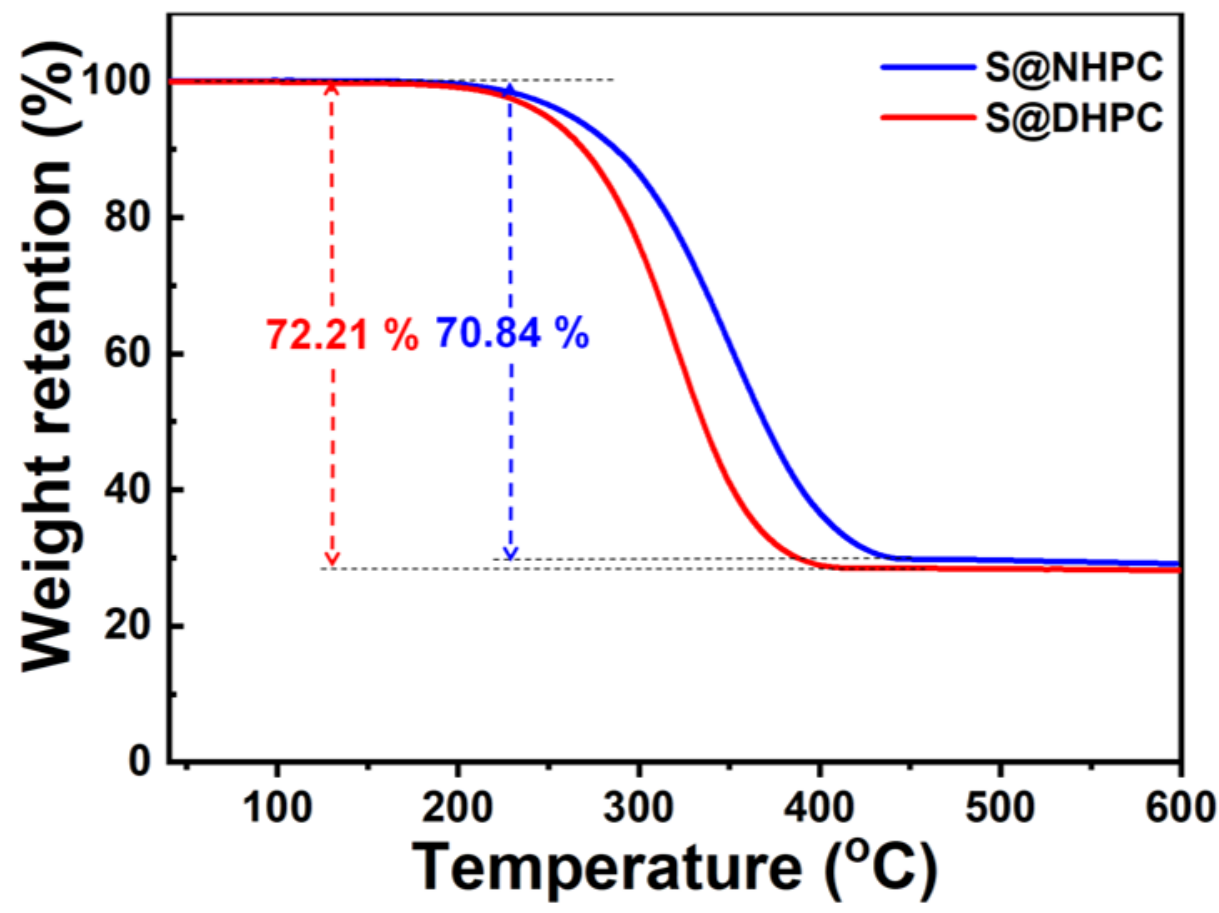

Figure S6. TGA curves of S@NHPC and S@DHPC. 


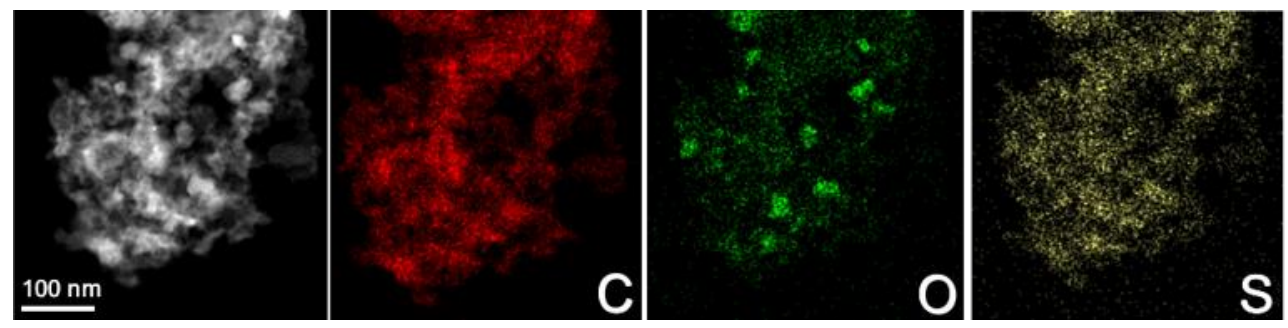

Figure S7. Elemental mapping of S@DHPC under TEM observation. 


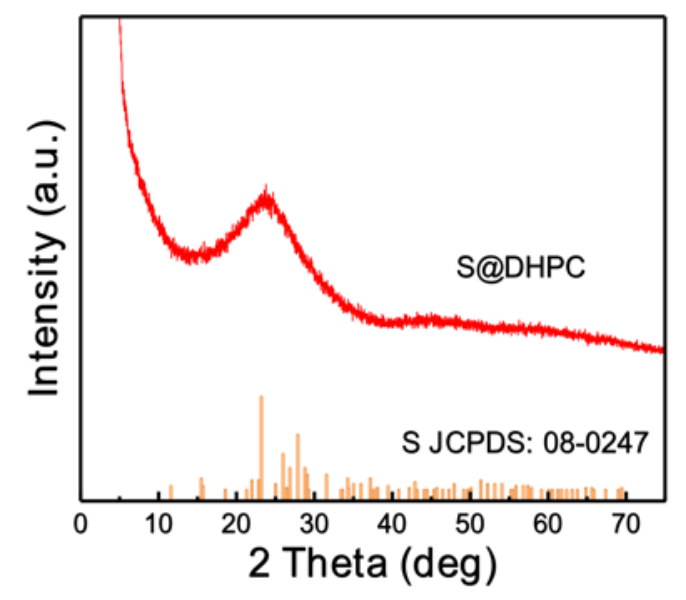

Figure S8. XRD pattern of S@ DHPC with a sulfur loading of 70 wt.\%. 


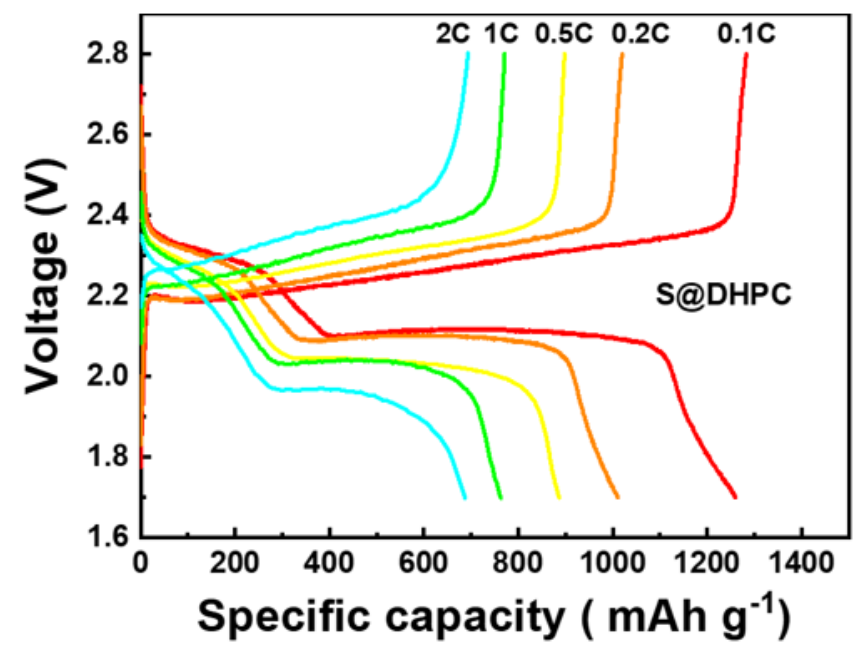

Figure S9. $1^{\text {st }}$ charge/discharge profiles of the S@DHPC cathode at various current rates between 0.1 and $2 \mathrm{C}$. 
Table S4. Comparison of electrochemical performance of S@DHPC with recently reported carbon hosts for Li-S batteries

\begin{tabular}{|c|c|c|c|c|c|}
\hline $\begin{array}{l}\text { Carbon-based sulfur } \\
\text { host }\end{array}$ & $\begin{array}{l}\text { Sulfur } \\
\text { content } \\
\text { (wt.\%) }\end{array}$ & $\begin{array}{c}\text { Sulfur } \\
\text { loading } \\
\left(\mathrm{mg} \mathrm{cm}^{-2}\right)\end{array}$ & $\begin{array}{c}\text { Initial } \\
\text { capacity } \\
\left(\mathrm{mAh}^{-1}\right)\end{array}$ & Retention & Ref. \\
\hline $\begin{array}{c}\text { N-doped porous } \\
\text { carbon/CNT hybrids }\end{array}$ & 62 & $\sim 1.5$ & $\sim 800$ at $0.5 \mathrm{C}$ & $\begin{array}{c}\sim 84 \% \text { after } 200 \\
\text { cycles }\end{array}$ & [1] \\
\hline $\begin{array}{l}\text { Hierarchical } \\
\text { structure } \\
\text { carbon fiber }\end{array}$ & 58 & $0.8-0.9$ & $\begin{array}{c}\sim 845 \text { at } 0.25 \\
\mathrm{C}\end{array}$ & $\begin{array}{c}\sim 77 \% \text { after } 100 \\
\text { cycles }\end{array}$ & {$[2]$} \\
\hline $\begin{array}{c}\text { N, O-codoped } \\
\text { hollow } \\
\text { carbon nanospheres }\end{array}$ & 66 & $\sim 1.4$ & $\begin{array}{c}\sim 1125 \text { at } 0.2 \\
\mathrm{C}\end{array}$ & $\begin{array}{c}\sim 74 \% \text { after } 200 \\
\text { cycles }\end{array}$ & [3] \\
\hline $\begin{array}{l}\text { EDA-functionalized } \\
\text { rGO }\end{array}$ & 60 & $\sim$ & $\begin{array}{c}\sim 770 \text { at } 0.5 \\
\mathrm{C}\end{array}$ & $\begin{array}{c}\sim 80 \% \text { after } 350 \\
\text { cycles }\end{array}$ & {$[4]$} \\
\hline $\begin{array}{c}\text { S@N-doped } \\
\text { graphitized carbon } \\
\text { spheres }\end{array}$ & 73 & $\sim 1.5$ & $\sim 857$ at $1 \mathrm{C}$ & $\begin{array}{c}\sim 70 \% \text { after } \\
150 \text { cycles }\end{array}$ & {$[5]$} \\
\hline $\mathrm{N}$-doped carbon & 60 & $0.8-1.0$ & $\begin{array}{c}\sim 1120 \text { at } 0.48 \\
\text { C }\end{array}$ & $\begin{array}{c}\sim 45 \% \text { after } 400 \\
\text { cycles }\end{array}$ & {$[6]$} \\
\hline $\begin{array}{c}\text { P-doped BN } \\
\text { nanosheets decorated } \\
\text { graphene }\end{array}$ & 60 & $\sim 1.0$ & $\begin{array}{c}\sim 1099 \text { at } 0.5 \\
\mathrm{C}\end{array}$ & $\begin{array}{c}\sim 58 \% \text { after } 500 \\
\text { cycles }\end{array}$ & [7] \\
\hline $\begin{array}{c}\text { Intrinsic } \\
\text { defect-enriched } \\
\text { hierarchically porous } \\
\text { carbon }\end{array}$ & 70 & $1.5-2.0$ & $\begin{array}{c}\sim 1180 \text { at } 0.5 \\
\mathrm{C}\end{array}$ & $\begin{array}{c}\sim 70 \% \text { after } 500 \\
\text { cycles }\end{array}$ & $\begin{array}{l}\text { This } \\
\text { work }\end{array}$ \\
\hline
\end{tabular}


(a)

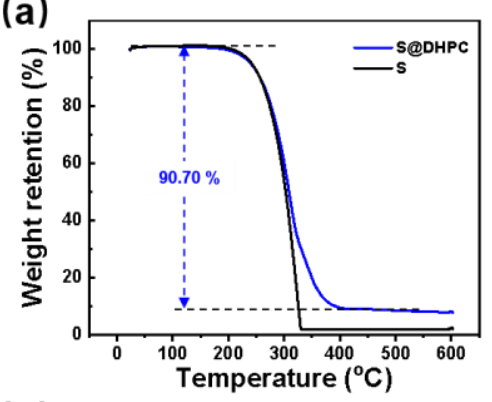

(d)

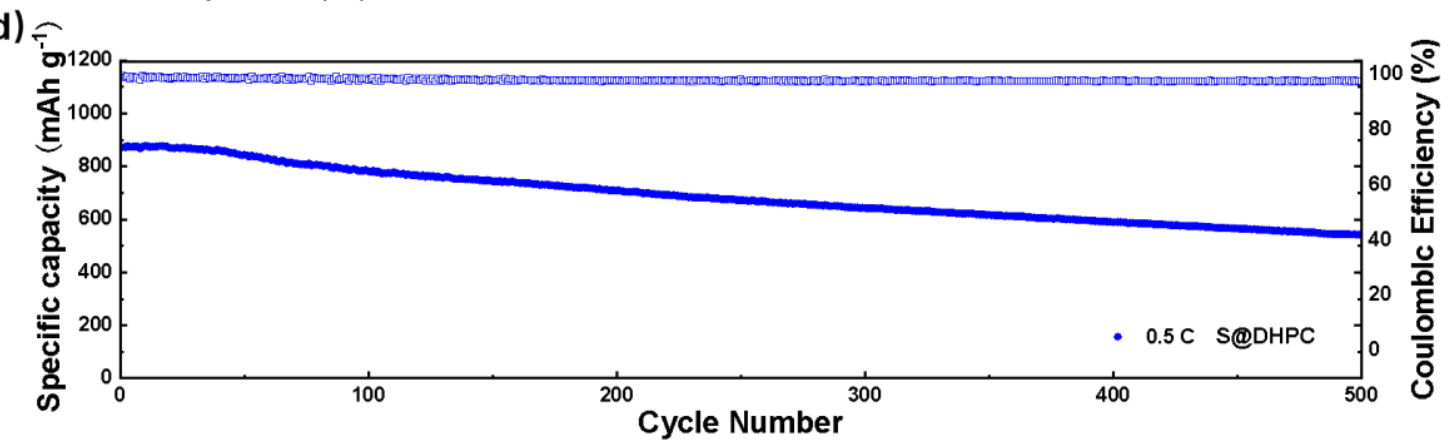

(c)
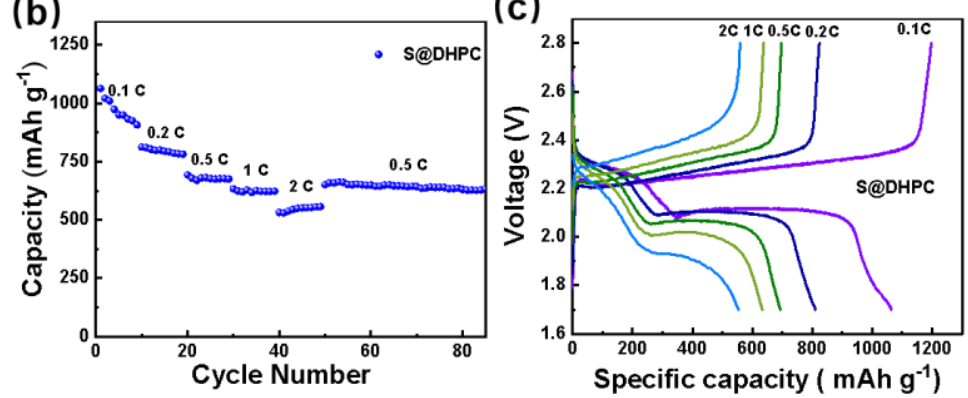

Figure S10. (a) TGA curves and (b) rate capability of S@DHPC with sulfur loading of 90 wt.\%; (c) $1^{\text {st }}$ charge/discharge profiles of S@DHPC cathode at various current rates between 0.1 and 2 C; (d) Long-term cycling performance of S@DHPC cathode at 0.5 C. 
(a)

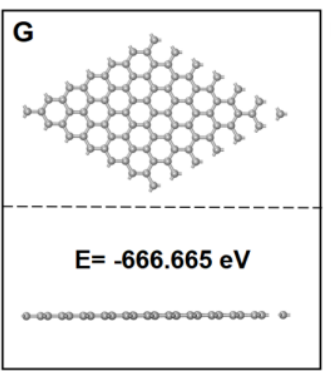

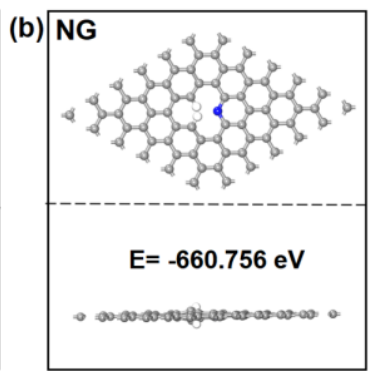
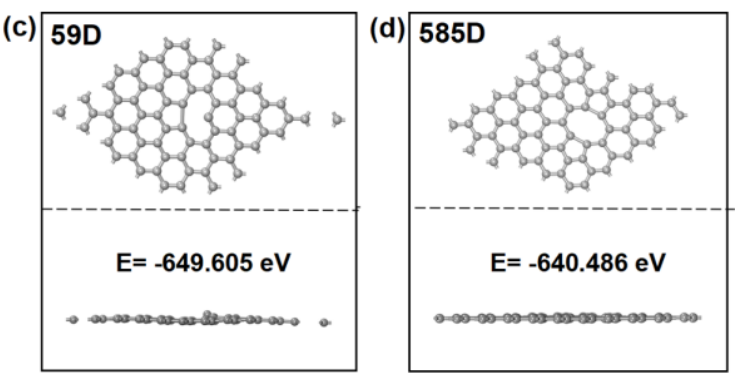

Figure S11. (a) Atomic structure of pristine graphene (G), (b) pyridinic N-doped graphene (NG), graphene with 5-9 defect (59D) (c) and 5-8-5 defect (585D) (d). The grey and blue balls represent $\mathrm{C}$ and $\mathrm{N}$ atoms, respectively. 


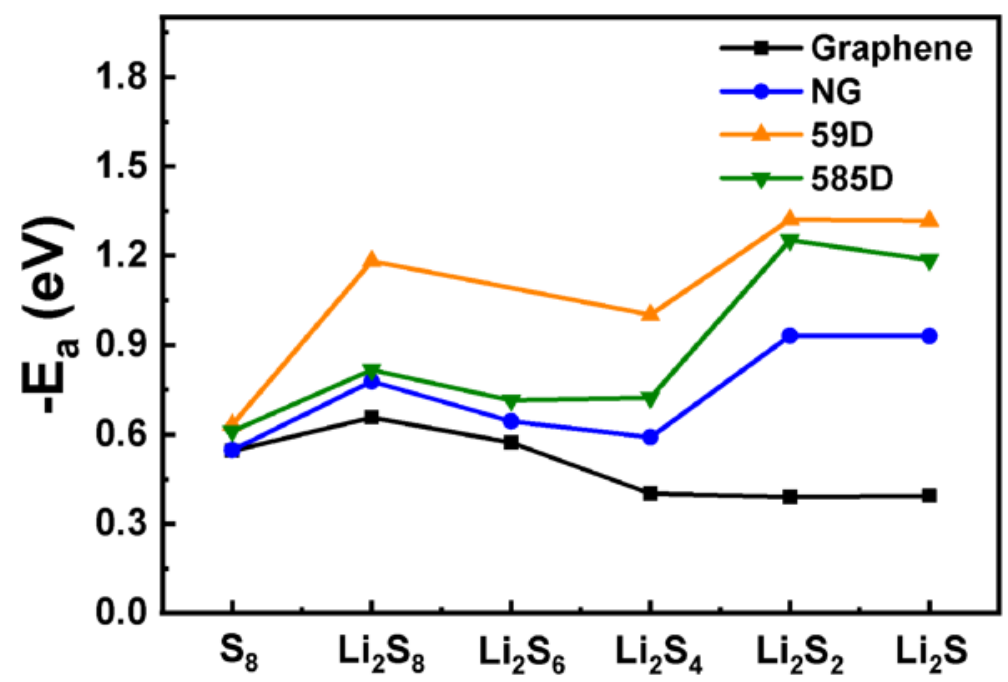

Figure S12. Adsorption energies for Li-S composites at six different lithation stages $\left(\mathrm{S}_{8}\right.$, $\mathrm{Li}_{2} \mathrm{~S}_{8}, \mathrm{Li}_{2} \mathrm{~S}_{6}, \mathrm{Li}_{2} \mathrm{~S}_{4}, \mathrm{Li}_{2} \mathrm{~S}_{2}, \mathrm{Li}_{2} \mathrm{~S}$ ) on different substrates. 
(a)

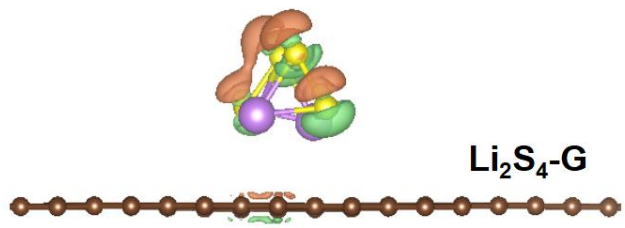

(c)

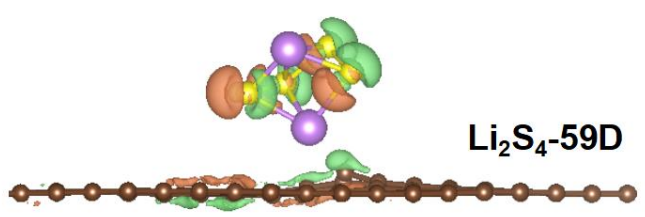

(b)

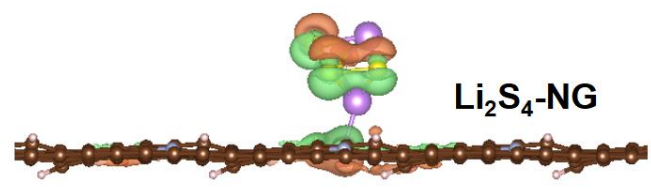

(d)

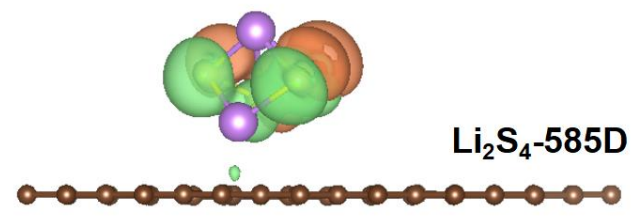

Figure S13. Charge density difference of $\mathrm{Li}_{2} \mathrm{~S}_{4}$ adsorbed on (a) G, (b) NG, (c) 59D, and (d) 585D. The charge density difference is expressed as $\Delta \rho=\rho\left(\operatorname{sub}+\operatorname{Li}_{2} \mathrm{~S}_{4}\right)-\rho(\mathrm{sub})-\rho\left(\mathrm{Li}_{2} \mathrm{~S}_{4}\right)$, where $\rho\left(\operatorname{sub}+\mathrm{Li}_{2} \mathrm{~S}_{4}\right), \rho(\mathrm{sub})$, and $\rho\left(\mathrm{Li}_{2} \mathrm{~S}_{4}\right)$ are the charge densities for adsorption system, substrates system, and Li-S cluster system, respectively. Here, green (brown) surfaces represent the spatial regions gain (loss) of charge. 


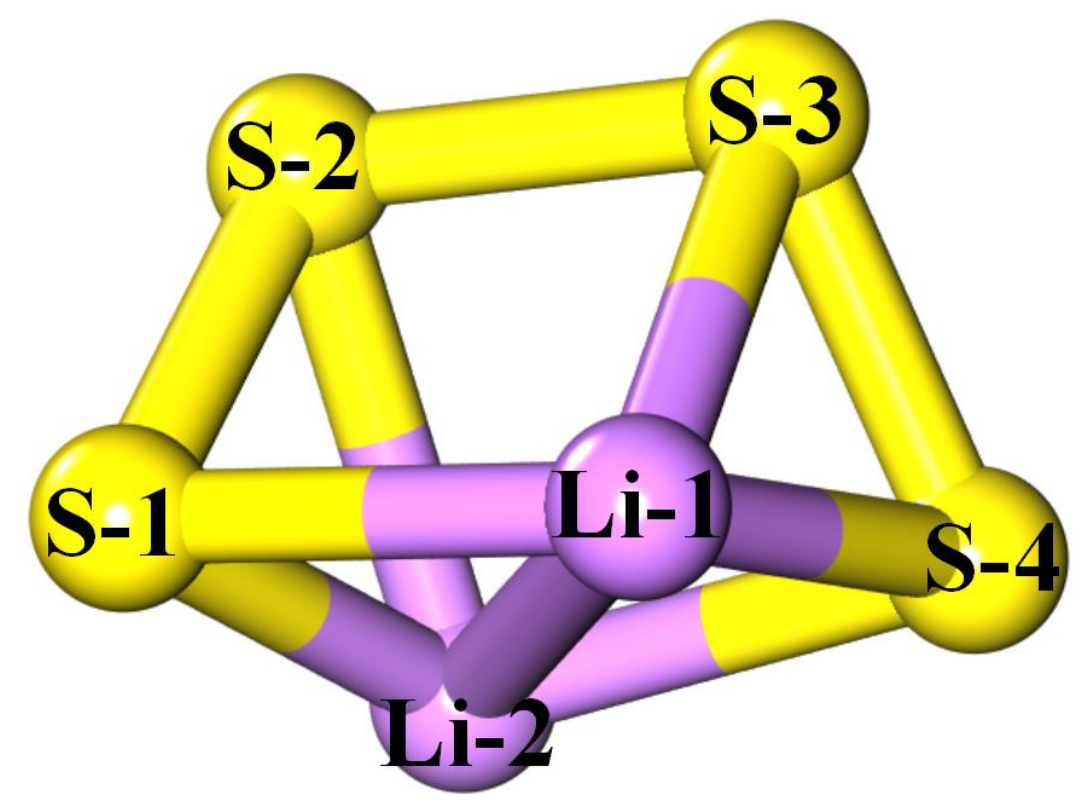

Figure S14. Molecule configuration of $\mathrm{Li}_{2} \mathrm{~S}_{4}$. Here, pink and yellow balls symbolize lithium and sulfur atoms.

Table S5. Bader charge difference of $\mathrm{Li}$ and $\mathrm{S}$ atoms of $\mathrm{Li}_{2} \mathrm{~S}_{4}$ after anchoring on the $\mathrm{G}, \mathrm{NG}$, 59D, and 585D. The unit is electron (e).

\begin{tabular}{ccccc}
\hline & $\mathrm{G}$ & $\mathrm{NG}$ & $59 \mathrm{D}$ & $585 \mathrm{D}$ \\
\hline$\Delta e_{\mathrm{Li}-1}$ & -0.86417 & -0.85021 & -0.84495 & -0.85647 \\
$\Delta e e_{\mathrm{Li}-2}$ & -0.86386 & -0.86014 & -0.86069 & -0.86234 \\
$\Delta e s-1$ & 0.68632 & 0.67474 & 0.65515 & 0.63446 \\
$\Delta e \mathrm{~S}-2$ & 0.15960 & 0.17212 & 0.15834 & 0.16398 \\
$\Delta e S-3$ & 0.15325 & 0.14391 & 0.11637 & 0.09779 \\
$\Delta e \mathrm{~S}-4$ & 0.69263 & 0.68706 & 0.62980 & 0.63899 \\
$\Delta e_{\mathrm{Li} 2 \mathrm{~S}} 4$ & -0.036232 & -0.032516 & -0.145978 & -0.183585 \\
\hline
\end{tabular}

There is bader charge difference inside Li-1 atom, Li-2 atom, S-1 atom, S-2 atom, S-3 atom, S-4 atom and $\mathrm{Li}_{2} \mathrm{~S}_{4}$ from top to bottom. 


\section{REFERENCES}

(1) Cai, J.; Wu, C.; Yang, S.; Zhu, Y.; Shen, P.; Zhang, K. Templated and Catalytic Fabrication of N-Doped Hierarchical Porous Carbon-Carbon Nanotube Hybrids as Host for Lithium-Sulfur Batteries. ACS Appl. Mater. Interfaces 2017, 9, 33876-33886.

(2) Wu, F.; Shi, L.; Mu, D.; Xu, H.; Wu, B. A Hierarchical Carbon Fiber/Sulfur Composite as Cathode Material for Li-S Batteries. Carbon, 2015, 86, 146-155.

(3) Peng, Y.; Zhang, Y.; Huang, J.; Wang, Y.; Li, H.; Hwang, B.; Zhao, J. Nitrogen and Oxygen Dual-Doped Hollow Carbon Nanospheres Derived from Catechol/Polyamine as Sulfur Hosts for Advanced Lithium Sulfur Batteries. Carbon 2017, 124, 23-33.

(4) Wang, Z.; Dong, Y.; Li, H.; Zhao, Z.; Wu, H. B.; Cao, C.; Liu, S.; Qiu, J.; Lou, X. W. Enhancing Lithium-Sulphur Battery Performance by Strongly Binding the Discharge Products on Amino-Functionalized Reduced Graphene Oxide. Nat. Commun. 2014, 5, 5002.

(5) Chen, Z.; Cheng, S.; Chen, Y.; Xia, X.; Liu, H. Pomegranate-Like S@N-Doped Graphitized Carbon Spheres as High-Performance Cathode for Lithium-Sulfur Battery, Mater. Lett., 2020, 263, 127283.

(6) Zhang, J.; Huang, M.; Xi, B.; Mi, K.; Yuan, A.; Xiong, S. Systematic Study of Effect on Enhancing Specific Capacity and Electrochemical Behaviors of Lithium-Sulfur Batteries. Adv. Energy Mater. 2018, 8, 1701330.

(7) Zhang, J.; Ma, W.; Feng, Z.; Wu, F.; Wei, D.; Xi B.; Xiong, S. P-Doped BN Nanosheets Decorated Graphene as the Functional Interlayer for Li-S Batteries. J. Energy Chem. 2019, $39,54-60$. 